\title{
Berufliche Atemwegssensibilisierungen durch Subtilisine
}

\section{V. van Kampen \\ R. Merget}

\author{
Occupational Airway Sensitization Due to Subtilisin
}

\section{Zusammenfassung}

Seit den 60er Jahren wurden zunehmend berufsbedingte Allergien gegen natürliche, neuerdings auch gegen modifizierte, rekombinant hergestellte Enzyme beobachtet. Besonders häufig industriell eingesetzt und deshalb auch häufig Auslöser von Atemwegssensibilisierungen sind Subtilisine. Die hier vorgestellte Auswertung klinischer Daten aus der Literatur belegt das Sensibilisierungspotential dieser Enzymgruppe. Durch eine größere Zahl von Studien sind Fälle von spezifischer Überempfindlichkeit der Atemwege durch Subtilisine gut belegt. Da Symptome, Hauttestergebnisse, Nachweis spezifischer IgE-Antikörper und Ergebnisse spezifischer Provokationstests in der Regel gut übereinstimmen, ist ein immunologischer Wirkmechanismus gesichert.

\section{Abstract}

Since the 1960s an increasing number of occupational allergies against natural, and against modified recombinant enzymes were observed. Subtilisin, a frequently used enzyme often has been shown to cause airway sensitization. The present evaluation of clinical literature data confirms the sensitization potential of subtilisin. Several cases of specific airway hyperresponsiveness caused by subtilisin are verified by a number of studies. As symptoms, results of skin prick tests, detection of specific IgEantibodies and results of specific bronchoprovocation tests are consistent, an immunologic mechanism is confirmed.

\section{Einleitung}

Serinproteasen bakteriellen Ursprungs (EC 3.4.21.62; früher EC 3.4.21.14) sind extrazelluläre Proteasen und werden nach dem bekanntesten Ursprungsorganismus Bacillus subtilis als Subtilisine bezeichnet [1]. Diese Enzyme spalten Eiweiße und dienen den Bakterien in erster Linie zur Nahrungsaufnahme. Die zwei wichtigsten Subtilisine (Alcalase ${ }^{\circledR}$ und Esperase ${ }^{\circledR}$ ) werden aus Bacillus subtilis bzw. B. licheniformis gewonnen. Einen Überblick über die häufigsten industriell eingesetzten Subtilisine gibt Tab.1. Proteasen, auch fungale, werden hauptsächlich in der Waschmittelund Reinigungsmittelindustrie verwendet. Weitere Märkte sind
Stärkeherstellung, Nahrungsmittelverarbeitung, Futtermittelund Silbergewinnung. Heutzutage werden Subtilisine häufig als Medikamente genutzt, die zur Entfernung von abgestorbenen Gewebeschichten und zur schnelleren Verheilung bei der Wundbehandlung eingesetzt werden. Das pH-Optimum der Alcalase ${ }^{\circledR}$ liegt im neutralen bis schwach alkalischen Bereich (pH 7-10), während die Esperase ${ }^{\circledast}$ unter stark alkalischen Bedingungen (bis pH 12) optimal arbeitet. Sie ist aber auch im schwächer alkalischen Milieu und bei niedrigen Temperaturen effektiv, was für die Waschmittelindustrie von besonderer Bedeutung ist [2]. 
Tab. 1 Kommerzielle Subtilisine, die in der Reinigungsmittelindustrie Anwendung finden (nach [2])

\begin{tabular}{|c|c|c|c|c|}
\hline Handelsname & Ursprungsorganismus & pH-Optimum & $\begin{array}{l}\text { Temperatur- } \\
\text { Optimum }\left({ }^{\circ} \mathrm{C}\right)\end{array}$ & Hersteller \\
\hline Alcalase & B. licheniformis & $8-9$ & 60 & Novo Nordisk, Dänemark \\
\hline Esperase & B. subtilis & $9-11$ & 60 & Novo Nordisk, Dänemark \\
\hline Savinase & B. subtilis & $9-11$ & 55 & Novo Nordisk, Dänemark \\
\hline Maxacal & B. subtilis & 11 & 60 & Gist-Brocades, Niederlande \\
\hline Maxatase & B. subtilis & $9,5-10$ & 60 & Gist-Brocades, Niederlande \\
\hline Opticlean & B. subtilis & $10-11$ & $50-60$ & Solvay Enzymes, Deutschland \\
\hline Optimase & B. subtilis & $9-10$ & $60-65$ & Solvay Enzymes, Deutschland \\
\hline Protosol & B. subtilis & 10 & 50 & Advan. Biochem., Indien \\
\hline Protease „Wuxi“ & B. subtilis & $10-11$ & $40-50$ & Wuxi Synder, China \\
\hline Proleather & B. subtilis & $10-11$ & 60 & Amano Pharm., Japan \\
\hline Durazym & veränderte Variante v. Savinase B. subtilis & $10-10,5$ & 50 & Novo Nordisk, Dänemark \\
\hline Maxapem & veränderte Variante aus B. subtilis & $11-12$ & 60 & Solvay Enzymes, Deutschland \\
\hline Purafect & $\begin{array}{l}\text { rekombinant; Donor: B. lentus exprimiert in: } \\
\text { B. subtilis }\end{array}$ & 10 & $40-65$ & Genecor Int., USA \\
\hline
\end{tabular}

Die folgende Literaturauswertung gibt darüber Auskunft, welche Relevanz bakteriellen Serinproteasen (Subtilisinen) hinsichtlich beruflicher Atemwegsallergien zukommt.

\section{Literaturauswertung}

Mit Hilfe geeigneter Stichwörter in sinnvoller Kombination wurden die Abstracts infrage kommender Veröffentlichungen in einer Datenbank (MEDLINE), die etwa 9 Millionen medizinische Literaturzitate umfasst, gefunden. Die Abstracts wurden durchgesehen und bei Bestätigung, dass es sich um geeignete Literatur zum Thema handelt, die Originalarbeiten besorgt. Uns so zugängliche Publikationen aus der Zeit zwischen 1969 und 2000 über Studien zur atemwegssensibilisierenden Wirkung von Subtilisinen, wurden hinsichtlich klinischer Daten ausgewertet. Erfasst wurden neben der Zahl der exponierten Personen die Prävalenzen der einzelnen Symptome bzw. Diagnosen. Darüber hinaus wurde festgehalten mit welchen Methoden (Hauttest, Bestimmung spez. IgE-Antikörper; Provokationstest) unter Verwendung welcher Testlösungen eine auf Subtilisine zurückzuführende Sensibilisierung diagnostiziert wurde. Einen zusammenfassenden Überblick über die Ergebnisse der Literaturauswertung gibt Tab 2.

\section{Ergebnisse}

Die atemwegssensibilisierende Wirkung von Subtilisin wurde bereits in den 60er Jahren beschrieben [3]. So berichtete Flindt 1969 [4] über 28 Beschäftigte in der Enzymindustrie, von denen 25 (89\%) über berufsbedingte Atemwegsbeschwerden klagten. Im Haut-Pricktest mit entsprechenden Enzymlösungen (Maxatase, Alcalase; $1 \mathrm{mg} / \mathrm{ml}$ ) zeigten 20 Probanden (71,4\%) bereits nach 5 min. eine deutliche Reaktion.

Auch Pepys u. Mitarb. [5] beschrieben bereits 1969 die Kasuistiken von drei in der Enzymproduktion Tätigen, die über bronchiale Beschwerden nach dem Umgang mit Bacillus subtilis-Enzymen klagten. In allen drei Fällen zeigten sich im Pricktest mit Alcalase- und Maxatase-Lösungen bei einer Konzentration von $10 \mathrm{mg} /$ $\mathrm{ml}$ sehr starke Reaktionen (negativ bei nicht-exponierten Kontrollprobanden). Bei zwei der Exponierten wurde der Hauttest mit schwächer konzentrierten Lösungen $(0,1 \mathrm{mg} / \mathrm{ml}$ bzw. $1 \mathrm{mg}$ / $\mathrm{ml}$ ) wiederholt und war ebenfalls positiv. Mit Hilfe von bronchialen Provokationstests (Alcalase-Lösungen 0,1 - $1 \mathrm{mg} / \mathrm{ml}$ ) konnten die Enzymsensibilisierungen bestätigt werden. Bei zwei Probanden traten duale Reaktionen, bei einem eine Spätreaktion auf.

Juniper u. Mitarb. [6] veröffentlichten 1977 die Ergebnisse einer 7-jährigen Längsschnittstudie (1968-1975) unter 1642 Exponierten einer englischen Waschmittelfabrik. Im Laufe der Studie wiesen 53 (3,2\%) der Beschäftigten respiratorische Beschwerden nach der Exposition gegenüber Bacillus subtilis-Proteasen auf. Bei 288 (17,5\%) der Exponierten konnte während dieses Zeitraums eine Reaktion im Haut-Pricktest mit Alcalase beobachtet werden. $81 \%$ der Hauttest-Positiven übten ihre Tätigkeit in Arbeitsbereichen mit hoher Enzymstaubkonzentration aus. Während der 7-jährigen Studie wurden 248 Seren exponierter Arbeiter im RAST auf das Vorhandensein Alcalase-spezifischer IgE-Antikörper getestet; dies führte in 64 Fällen (26\%) zu einem positiven Resultat, wobei eine gute Korrelation zwischen den Hauttest- und den RAST-Ergebnissen bestand.

Newhouse u. Mitarb. [7] berichteten über ihre Studie unter 271 Mitarbeitern einer britischen Reinigungsmittelfirma. Im HautPricktest mit einer 1 bzw. 5\%igen Alcalase-Lösung ergab sich bei 57 (21\%) der Probanden ein positives Ergebnis. Von diesen klagten 42 (74\%) über Atemwegsbeschwerden und weitere 10 (17,5\%) über nasale Irritationen. Von den 214 im Hauttest Negativen gaben jedoch auch 75 (35\%) Atemwegsbeschwerden an.

121 Mitarbeiter einer britischen Waschmittelfirma nahmen an einer Studie teil, nachdem fünf Angestellte an respiratorischen Beschwerden nach der Exposition gegen Bacillus subtilis-Enzyme litten [8]. Insgesamt gaben 77 (64\%) der Exponierten Rhinitis/ Konjunktivis, 17 (14\%) Husten und 41 (34\%) Atemwegsbeschwerden im Zusammenhang mit der Enzymstaubexposition 
Tab. 2 Klinische Untersuchungen, die die atemwegssensibilisierende Wirkung von Subtilisin belegen

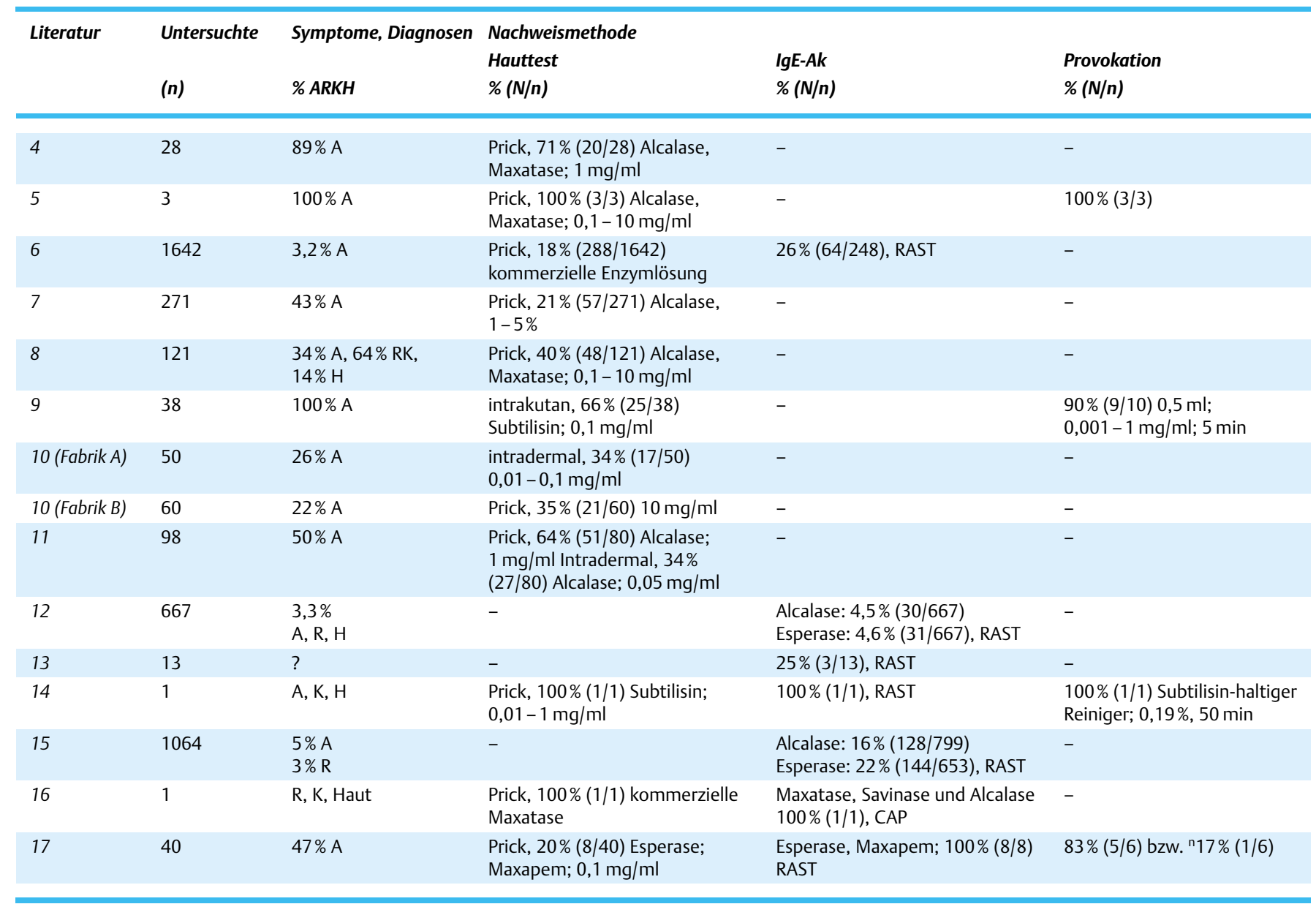

Abkürzungen und Fußnoten: \% Prozent der Positiven (N/n absolute Zahl der pos. Reaktionen bezogen auf die Zahl der Untersuchten) arbeitsplatzbezogene Symptome: ARKH Atemwegsbeschwerden und/oder Rhinitis und/oder Konjunktivitis und/oder Husten Bei der Provokation handelt es sich um bronchiale Provokationen, wenn nicht durch n'(nasale Provokation) indiziert

an. Im Haut-Pricktest ergab sich, dass $64 \%$ (16 v. 25) der Atopiker, jedoch lediglich $33 \%$ (32 v. 96) Nicht-Atopiker eine positive Reaktion auf Alcalase und/oder Maxatase $(0,1-10 \mathrm{mg} / \mathrm{ml})$ zeigten. Im Lungenfunktionstest wurde bei 21 von 48 (44\%) Sensibilisierten, jedoch nur bei 10 von 73 (14\%) Nicht-Sensibilisierten eine Erniedrigung von $\mathrm{FEV}_{1} / \mathrm{FVC}$ registriert.

Etwa vier Jahre nach dem erfolgreichen Einsatz von Proteasen in der Reinigungsmittelherstellung in Europa fanden die Enzyme auch in den USA Anwendung. Franz u. Mitarb. [9] berichten, dass ca. ein Jahr nach Beginn der Verwendung von Proteasen aus B. subtilis bzw. B. licheniformis in einem amerikanischen Betrieb zur Herstellung von Reinigungsmitteln einige Arbeiter in Verbindung mit der Enzymstaubexposition über Beschwerden an den oberen und unteren Atemwegen klagten. In ihrer Studie untersuchten sie 38 der symptomatischen Beschäftigten. Im Intrakutantest unter Verwendung von jeweils $0,02 \mathrm{ml}$ der entsprechenden Subtilisinlösungen $(0,1 \mathrm{mg} / \mathrm{ml}$ PBS, pH 7,2) zeigten 25 Personen (66\%) nach 15 - 20 min eine positive Reaktion. Der passive Transfer der Seren von fünf im Hauttest deutlich positiven Probanden auf nicht-exponierte Kontrollpersonen führte in allen Fällen nach 48 Stunden zu einem positiven Intrakutantest bei den Kontrollen. Die Reaktionen blieben aus, wenn die transferierten Seren zuvor $30 \mathrm{~min}$. lang auf $56^{\circ} \mathrm{C}$ erhitzt worden waren.
Präzipitierende Subtilisin-spezifische Antikörper waren mittels Ouchterlony-Test bei $48 \%$ von 25 exponierten und bei $29 \%$ von 41 nicht-exponierten Personen nachweisbar. Im bronchialen Provokationstest ( $5 \mathrm{~min} ., 0,5 \mathrm{ml}$ vernebelte Subtilisinlösung, Konz.: 0,001-1 mg/ml) trat bei 9 von 10 getesteten symptomatischen Personen (90\%) eine Soforttypreaktion auf, wohingegen die Provokationen der 5 Kontrollpersonen negativ verlief.

Ebenfalls 1971 wurden die Ergebnisse einer Studie über Enzymstaubexponierte in zwei amerikanischen Waschmittelfabriken publiziert [10]. Von 125 Beschäftigten der Fabrik A nahmen 50, von 486 Beschäftigten der Fabrik B 60 an der Studie teil. Von den Studienteilnehmern, die jeweils in drei Expositionsgruppen (hoch, mittel, niedrig) eingeteilt wurden, klagten $26 \%$ (A) bzw. $22 \%$ (B) über respiratorische Beschwerden. Hauttests wurden in Fabrik A intradermal $(0,01-0,1 \mathrm{mg} / \mathrm{ml})$, in Fabrik B als Pricktest $(10 \mathrm{mg} / \mathrm{ml})$ durchgeführt. In beiden Fällen zeigte sich eine auffallend hohe, dosisabhängige Prävalenz. So waren in Fabrik A in der hochexponierten Gruppe 9 von 20 (45\%), in der mittelexponierten Gruppe 8 von 15 (53\%), in der niedrigexponierten Gruppe dagegen kein Test positiv. In Fabrik B zeigten 11 von 21 (52\%) Personen der hochexponierten Gruppe, 7 von 20 (35\%) in der mittelexponierten Gruppe und 3 von 19 (16\%) in der niedrigexponierten Gruppe im Hauttest ein positives Ergebnis. 
Mitchell und Gandevia [11] publizierten 1971 die Ergebnisse einer Querschnittstudie in einer Reinigungsmittelfirma. 49 der 98 (50\%) Studienteilnehmer klagten über Beschwerden im unteren Respirationstrakt. Im Haut-Pricktest mit Alcalase $(1 \mathrm{mg} / \mathrm{ml})$ zeigten 51 von $80(64 \%)$, im Intrakutantest $(0,05 \mathrm{mg} / \mathrm{ml}) 27$ von 80 (34\%) eine positive Reaktion. Lediglich zwei der im Intrakutantest Positiven waren im Pricktest negativ.

In einer 1981 veröffentlichten skandinavischen Längsschnittstudie [12] wurden insgesamt 667 in der Subtilisin-Produktion (Esperase und Alcalase) Tätige untersucht. Im Verlauf der 10jährigen Studie (1970 - 1980) klagten 3,3\% der Beschäftigten über arbeitsplatzbezogene Beschwerden wie Rhinitis, Husten und Atemnot. Esperase-spezifische IgE-Antikörper konnten bei 31 Exponierten (4,6\%) mittels RAST nachgewiesen werden. Mit einer Ausnahme wiesen diese Personen auch IgE-Antikörper gegen Alcalase auf.

Ob auch die seit einiger Zeit in der Waschmittelindustrie eingesetzten granulierten Bacillus-Enzyme atemwegssensibilisierend wirken, sollte in einer amerikanischen Studie mit 13 exponierten, 2 früher exponierten und 9 nicht-exponierten Beschäftigten untersucht werden [13]. Mittels RAST wurden bei 3 (25\%) exponierten Probanden spezifische IgE-Antikörper gegen Bacillus-Enzyme nachgewiesen, jedoch bei keinem der früher exponierten bzw. der Nicht-Exponierten.

In einem Fallbericht beschreiben Lemiere u. Mitarb. [14] einen 41jährigen Angestellten, der im Rahmen seiner beruflichen Tätigkeit medizinische Geräte mittels einer Subtilisin-haltigen Lösung (Klenzyme) reinigte. Etwa ein Jahr nach Arbeitsaufnahme entwickelte er am Arbeitsplatz Husten, Konjunktivitis und Atemwegsbeschwerden; an den Wochenenden und während des Urlaubs war er beschwerdefrei. Im Provokationstest mit Methacholin kam es zu einem signifikanten $\mathrm{FEV}_{1}$-Abfall im Sinne eines hyperreaktiven Bronchialsystems. Im Haut-Pricktest wurden Subtilisinlösungen verschiedener Konzentrationen $(0,01-1 \mathrm{mg} / \mathrm{ml})$ eingesetzt, die alle zu einem positiven Ergebnis führten. Hinsichtlich Subtilisin-spezifischer IgE-Antikörper im Serum des Patienten wurde mittels RAST ein Bindungswert von 31,7\% erzielt (Werte ab 5,7\%=positiv). In einem mit dem relevanten Subtilisin-haltigen Reiniger (0,19\%ige Lösung über 50 min.) durchgeführten spezifischen bronchialen Provokationstest kam es zu einer ungewöhnlichen isolierten Spätreaktion. Nach 4 Stunden war der maximale $\mathrm{FEV}_{1}$-Abfall von $25 \%$ erreicht.

In einer retrospektiven Follow-up-Studie werteten Johnsen u. Mitarb. [15] die Daten von 1064 in der Enzymproduktion Tätigen aus. Innerhalb von 3 Jahren klagten 5\% der Beschäftigten über asthmatische und $3 \%$ über rhinitische Beschwerden, in einigen Fällen auch über Urtikaria in Verbindung mit der Enzymstaubexposition am Arbeitsplatz. Spezifische IgE-Antikörper (RAST) gegen Alcalase wiesen $16 \%$ von 799, gegen Esperase $22 \%$ von 653 Getesteten auf. Haut- und Provokationstests wurden nicht durchgeführt.

Über Beschwerden (Rhinokonjunktivitis, Urtikaria) beim Umgang mit pulverisierten Bacillus subtilis-Enzymen bei einer Laborassistentin berichten Kempf u. Mitarb. [16]. Der Pricktest mit kommerzieller Maxatase-Lösung verlief ebenso wie der Nach- weis spezifischer IgE-Antikörper (CAP-Test) gegen Maxatase, Savinase und Alcalase positiv.

Eine Querschnittuntersuchung unter 40 exponierten Beschäftigten einer Waschmittelfirma und 36 nicht-exponierten Kontrollen führten Vanhanen u. Mitarb. durch [17]. Von den Exponierten gaben bei einer Befragung $47 \%$, von den Nicht-Exponierten $11 \%$ respiratorische Beschwerden am Arbeitsplatz an. Im Haut-Pricktest (Proteinkonzentration $0,1 \mathrm{mg} / \mathrm{ml}$ ) zeigten 8 Exponierte $(20 \%)$, jedoch keiner der Kontrollprobanden eine Reaktion auf die Bacillus subtilis-Proteasen Esperase und Maxapem. In allen Seren der Hauttest-Positiven, die unter arbeitsplatzbezogener Rhinitis ( $\mathrm{n}=8)$ bzw. Asthma $(\mathrm{n}=1)$ litten, konnten mittels RAST spezifische IgE-Antikörper nachgewiesen werden. In 5 von 6 Fällen wurde die Proteasesensibilisierung durch eine spezifische nasale, in einem Fall durch eine bronchiale Provokation bestätigt.

In einem Meerschweinchenmodell konnte gezeigt werden, dass sowohl bei passiver kutaner Anaphylaxie als auch bei inhalativer Exposition signifikant höhere Antikörper-Titer gegen die ebenfalls in der Reinigungsmittelindustrie verwendeten Enzyme $\alpha$-Amylase und Lipase induziert wurden, wenn man diese zusammen mit einer Protease (z. B. Alcalase, Savinase) verabreichte [18].

\section{Schllussfolgerung}

Durch eine größere Zahl von Studien sind Fälle von spezifischer Überempfindlichkeit der Atemwege oder Lunge durch bakterielle Proteasen (Subtilisine) gut belegt (Übersicht s. Tab. 2). Da Symptome, Hauttestergebnisse, der Nachweis spezifischer IgEAntikörper und die Ergebnisse spezifischer Provokationsteste in der Regel gut übereinstimmen, ist ein immunologischer Wirkmechanismus gesichert. Auch die in der letzter Zeit bevorzugt eingesetzten granulierten Enzympräparate scheinen keinen verlässlichen Schutz vor einer Atemwegssensibilisierung zu bieten. Zusätzlich geben tierexperimentelle Daten Anlass zu der Vermutung, dass die Inhalation von Protease-haltigen Stäuben die atemwegssensibilisierende Wirkung anderer in der Reinigungsmittelindustrie eingesetzter Enzyme verstärkt.

\section{Literatulr}

${ }^{1}$ Baur X, Sander I, van Kampen V. Aerogene Eyzyme sind aggressive berufliche Inhalationsallergene. Dtsch Med Wochenschr 2000; 125: 912-917

2 Ganesh Kumar C, Malik RK, Tiwari MP. Novel enzyme-based detergents: An Indian perspective. Curr Sci 1998; 75: 1312-1319

3 Wüthrich B, Ott F. Occupational asthma due to proteases in the detergent industry. Schweiz Med Wochenschr 1969; 99: 1584-1586

${ }^{4}$ Flindt ML. Pulmonary disease due to inhalation of derivatives of Bacillus subtilis containing proteolytic enzyme. Lancet 1969; 1: 1177-1181

${ }^{5}$ Pepys J, Longbottom JL, Hargreave FE, Faux J. Allergic reactions of the lungs to enzymes of Bacillus subtilis. Lancet 1969; 1: 1181-1184

${ }^{6}$ Juniper CP, How MJ, Goodwin BF, Kinshott AK. Bacillus subtilis enzymes: a 7-year clinical, epidemiological and immunological study of an industrial allergen. J Soc Occup Med 1977; 27: 3-12

${ }^{7}$ Newhouse ML, Tagg B, Pocock SJ, McEwan AC. An epidemiological study of workers producing enzyme washing powders. Lancet 1970; 1 : 689-693

${ }^{8}$ Greenberg M, Milne JF, Watt A. Survey of workers exposed to dusts containing derivatives of Bacillus subtilis. Br Med J 1970; 2: 629-633 
${ }^{9}$ Franz T, McMurrain KD, Brooks S, Bernstein IL. Clinical, immunologic, and physiologic observations in factory workers exposed to $\mathrm{B}$. subtilis enzyme dust. J Allergy 1971; 47: 170-180

${ }^{10}$ Weill H, Waddell LC, Ziskind M. A study of workers exposed to detergent enzymes. JAMA 1971; 217 (4): 425-433

${ }^{11}$ Mitchell CA, Gandevia B. Respiratory symptoms and skin reactivity in workers exposed to proteolytic enzymes in the detergent industry. Am Rev Respir Dis 1971; 104: 1-12

12 Zachariae H, Hoech-Thomsen J, Witmeur O, Wide L. Detergent enzymes and occupational safety. Observations on sensitization during Esperase production. Allergy 1981; 36: 513-516

${ }^{13}$ Liss GM, Kominsky JR, Gallagher JS, Melius J, Brooks SM, Bernstein IL. Failure of enzyme encapsulation to prevent sensitization of workers in the dry bleach industry. J Allergy Clin Immunol 1984; 73: 348-355

${ }^{14}$ Lemiere C, Cartier A, Dolovich J, Malo JL. Isolated late asthmatic reaction after exposure to a high-molecular-weight occupational agent, subtilisin. Chest 1996; 110: $823-824$
${ }^{15}$ Johnsen CR, Sorensen TB, Ingemann Larsen A, Bertelsen Secher A, Andreasen E, Kofoed GS, Fredslund Nielsen L, Gyntelberg F. Allergy risk in an enzyme producing plant: a retrospective follow-up study. Occup Environ Med 1997; 54: 671 - 675

${ }^{16} \mathrm{Kempf}$ W, Oman H, Wuthrich B. Allergy to proteases in medical laboratory technicians: A new occupational disease? J Allergy Clin Immunol 1999; 104: 700-701

${ }^{17}$ Vanhanen M, Tuomi T, Tiikkainen U, Tupasela O, Voutilainen R, Nordman H. Risk of enzyme allergy in the detergent industry. Occup Environ Med 2000; 57: 121 - 125

18 Sarlo K, Ritz HL, Fletcher ER, Schrotel KR, Clark ED. Proteolytic detergent enzymes enhance the allergic antibody responses of guinea pigs to nonproteolytic detergent enzymes in a mixture: implications for occupational exposure. J Allergy Clin Immunol 1997; 100: 480-487 